\title{
Single nucleotide polymorphisms and microsatellites in the canine glutathione S-transferase pi 1 (GSTP1) gene promoter
}

James Sacco * (D), Sarah Mann and Keller Toral

\begin{abstract}
Background: Genetic polymorphisms within the glutathione S-transferase P1 (GSTP1) gene affect the elimination of toxic xenobiotics by the GSTP1 enzyme. In dogs, exposure to environmental chemicals that may be GSTP1 substrates is associated with cancer. The objectives of this study were to investigate the genetic variability in the GSTP1 promoter in a diverse population of 278 purebred dogs, compare the incidence of any variants found between breeds, and predict their effects on gene expression. To provide information on ancestral alleles, a number of wolves, coyotes, and foxes were also sequenced.

Results: Fifteen single nucleotide polymorphisms (SNPs) and two microsatellites were discovered. Three of these loci were only polymorphic in dogs while three other SNPs were unique to wolves and coyotes. The major allele at c.-46 is T in dogs but is $C$ in the wild canids. The c.-185 delT variant was unique to dogs. The microsatellite located in the $5^{\prime}$ untranslated region ( $\left.5^{\prime} \mathrm{UTR}\right)$ was a highly polymorphic GCC tandem repeat, consisting of simple and compound alleles that varied in size from 10 to 22 -repeat units. The most common alleles consisted of 11, 16, and 17-repeats. The 11-repeat allele was found in 10\% of dogs but not in the other canids. Unequal recombination and replication slippage between similar and distinct alleles may be the mechanism for the multiple microsatellites observed. Twenty-eight haplotypes were constructed in the dog, and an additional 8 were observed in wolves and coyotes. While the most common haplotype acrossbreeds was the wild-type ${ }^{*} 1 \mathrm{~A}(17)$, other prevalent haplotypes included *3A(11) in Greyhounds, *6A(16) in Labrador Retrievers, *9A(16) in Golden Retrievers, and *8A(19) in Standard Poodles. Boxers and Siberian Huskies exhibited minimal haplotypic diversity. Compared to the simple $16^{*} 1$ allele, the compound $16^{*} 2$ allele (found in $12 \%$ of dogs) may interfere with transcription factor binding and/or the stability of the GSTP1 transcript.
\end{abstract}

Conclusions: Dogs and other canids exhibit extensive variation in the GSTP1 promoter. Genetic polymorphisms within distinct haplotypes prevalent in certain breeds can affect GSTP1 expression and carcinogen detoxification, and thus may be useful as genetic markers for cancer in dogs.

Keywords: GSTP1, Promoter, Canine, Polymorphism, Microsatellite, Breed, Carcinogen

\section{Background}

Glutathione S-transferases (GSTs) are a widely-expressed family of enzymes that play an important role in detoxification by catalyzing the conjugation of many hydrophobic and electrophilic compounds with the reduced form of the tripeptide glutathione (GSH). Substrates for these enzymes include an extensive array of xenobiotics, including

\footnotetext{
* Correspondence: james.sacco@drake.edu

Ellis Pharmacogenomics Laboratory, College of Pharmacy and Health Sciences, Drake University, Des Moines, IA 50311, USA

drugs, toxins and environmental carcinogens [1]. The conjugation of xenobiotics with GSH is catalyzed by GSTs in the cytosol, endoplasmic reticulum and mitochondria. Based on their biochemical, immunologic, and structural properties, the cytosolic or soluble GSTs are categorized into 4 main classes: alpha (A), mu (M), pi (P), and theta (T). Each class can be subcategorized into various related isozymes.

GST pi 1 (GST-P1) is expressed in extrahepatic tissues such as placenta, lung, gut and erythrocytes [2,3], where it is involved in the biotransformation of carcinogens 
such as acrolein [4], and benzo[a]pyrene diol epoxide (BPDE) [5], endocrine-disrupting pesticides such as atrazine [6], and numerous drugs, including cisplatin [7] and chlorambucil [8]. While humans and rats express one subunit belonging to the P1 class of GSTs, GSTP1, mice express two pi subunits, GSTP1 and GSTP2. The situation may be analogous in dogs, who appear to have one annotated GSTP1 gene, located on chromosome 18 (chr18:49,905,161-49,908,182 on the CanFam3 dog genome assembly), and another uncharacterized 'GSTP-like' gene (chr18:49,950,873-49,953,818) adjacent to it.

Polymorphisms in the GSTP1 gene can lead to impaired GST function. These DNA variants influence susceptibility to carcinogens and the toxic effects of some pesticides, affect the response to chemotherapy used in the treatment of metastatic colon cancer and multiple myeloma, and modify the risk of several forms of cancer. For example, a single nucleotide polymorphism (SNP) in the coding region of human GSTP1 (c.313 A > G, denoted as rs1695), present in $35 \%$ of the population, leads to decreased enzyme activity $[9,10]$. This polymorphism results in a Ile105Val change in the GSTP1 enzyme that is, for example, less able to conjugate carcinogens such as BPDE [11]. In fact, this allelic variant has been associated with increased susceptibility to acute myeloid leukemia, prostate, lung, bladder, gastric, oral and breast cancer [12-18]. Genetic and epigenetic variability in the promoter region of GSTP1 also affects gene expression and influences susceptibility to several types of cancer [19-22].

In dogs (Canis lupus familiaris), several studies suggest a possible causal relationship between exposure to certain environmental chemicals and canine cancers. An increased risk of canine malignant lymphoma has been associated with owner application or commercial treatment of lawns with the commonly used herbicide 2,4-dichlorophenoxyacetic acid [23, 24], as well as heterogeneous pesticide mixtures [25]. Living in industrial areas, or in proximity to polluted sites, incinerators, and/or radioactive waste, are also significant risk factors $[26,27]$. The expression of GSTs in dogs can vary widely. GST-T activities have been characterized in livers from research beagles, and $12 \%$ of these dogs had virtually no GST-T protein expression or activities [28]. In addition, GSTT1 polymorphisms have been associated with lymphoma in dogs [29]. It is not known if the expression of GSTP1 shows a similarly wide variability between individual dogs, or whether GSTP1-null allelic variants exist in the population. A preliminary study we have conducted (unpublished data) indicated that, in contrast to the coding region, the canine GSTP1 promoter sequence contained multiple polymorphisms. By affecting gene expression, these polymorphisms could potentially impact detoxification, and consequently modulate the risk of cancer and other diseases in dogs.

Therefore, the objective of this study was to investigate the variation in the GSTP1 gene promoter in a diverse population of domestic dogs, compare the incidence of these variants between dog breeds, and predict the potential functional effects of these genetic variants. In order to determine which polymorphisms may have been introduced by domestication or breed formation, we also studied the same genetic region in other species representing the Canidae family, the gray wolf (Canis lupus), coyote (Canis latrans), red fox (Vulpes vulpes) and gray fox (Urocyon cinereoargenteus).

\section{Methods}

\section{Animals and sample collection}

The 278 unrelated pure-bred dogs (144 males, 134 females; 102 breeds) recruited for this study were either privately owned or provided through the collaboration of Paws \& Effect, the Des Moines Kennel Club, and the Des Moines Obedience and Training Club (Additional file 1). Genomic DNA from 37 of these dogs was kindly donated by Lauren Trepanier, University of WisconsinMadison, and Michael Court, Washington State University. No dogs were euthanized for the purpose of this study. The American grey wolves $(n=14)$, coyote $(n=7)$ and foxes $(n=8)$ were either privately owned or from the Colorado Wolf and Wildlife Center (Divide, CO), Wolf Park (Battle Ground, IN), Blanke Park Zoo (Des Moines, IA), and the JAB Canid Education and Conservation Center (Santa Ysabel, CA). Buccal cell samples were collected from the dogs using cheek swabs designed for use in canines (Performagene ${ }^{\circ}$, DNA Genotek Inc.). DNA collected in this manner is stable for at least one year when stored at room temperature. All experimental procedures were approved by the Drake University Institutional Animal Care and Use Committee.

\section{DNA sequencing}

Following inactivation of nucleases and precipitations of impurities in the buccal samples, genomic DNA was purified via ethanol precipitation. We used $200 \mathrm{ng}$ of this DNA in a $25 \mu \mathrm{l}$ of reaction mixture containing $0.25 \mu \mathrm{M}$ of each primer (F: CCACCTCCCTCCTTCC AGTA; R: GCCTTCCAGGAACTCTGACC), $1 \mu \mathrm{l}$ of GC Enhancer, and $12.5 \mu \mathrm{L}$ of Amplitaq Gold 360 Master Mix (Applied Biosystems, Foster City, CA) in order to amplify the genomic region from c.-619 to c. $1+123$ ). After an initial incubation at $95^{\circ} \mathrm{C}$ for $10 \mathrm{~min}$, PCR amplification was performed for 40 cycles consisting of $95^{\circ} \mathrm{C}$ for $30 \mathrm{~s}, 57^{\circ} \mathrm{C}$ for $30 \mathrm{~min}$ and $72{ }^{\circ} \mathrm{C}$ for $45 \mathrm{~s}$, followed by a final extension at $72{ }^{\circ} \mathrm{C}$ for $7 \mathrm{~min}$. The specificity of each PCR was checked by electrophoresis on a $1.5 \%$ agarose gel. Following purification by Exo-SapIT (Affymetrix, 
Santa Clara, CA), the amplicons were submitted to bidirectional Sanger sequencing using the Big-Dye Terminator v3.1 (Eurofins Genomics, Louisville, KY). Five percent of the samples were randomly chosen and resequenced to confirm the initial genotype result. Sequence assembly and identification of genetic polymorphisms was performed using Staden package software (http://staden.sourceforge.net/).

\section{Data analysis}

Haploview [30] was used to calculate population genetic descriptors as well as to infer linkage disequilibrium (LD) parameters ( $\mathrm{D}^{\prime}$ and logarithm of odds scores, LOD). The chi-squared test for independence was used to assess Hardy-Weinberg equilibrium. Interallelic LD between multiallelic loci and SNPs was estimated by MIDAS software [31]. This program was also used to infer evolutionary relationships between the polymorphisms found.

Multiple comparative DNA sequence alignments between canine and other mammalian GSTP1 promoter and transcript sequences was performed using Clustal Omega [32] in order to determine the degree to which the sequences are conserved. Pairwise sequence alignment was computed by PromoterWise [33], which allows for inversions and translocations that are common in promoters. The aligning of transcription factors to the canine GSTP1 promoter sequence was performed by using TRANSFAC position-specific scoring matrix models through the LASAGNA algorithm $\mathrm{v} 2.0$, an integrated webtool for transcription factor binding site search and visualization [34]. By inputting sequence variants, we identified the effects that promoter polymorphisms may have on proteins that may bind to our sequence of interest. RNA secondary structure prediction was carried out using the Mfold program v 3.5 [35]. This program determines optimal and suboptimal secondary structures of RNA calculated for $1 \mathrm{M} \mathrm{NaCl}$ at $37{ }^{\circ} \mathrm{C}$, and computes free energy contributions for various potential secondary structures. Estimation of repeat variability (VARscores) within microsatellite sequences was determined by SERV. Larger VARscores correlate with higher predicted repeat variability [36].

\section{Results}

Fourteen polymorphic loci, including twelve SNPs and two microsatellites were found in the proximal GSTP1 promoter region (chr18: 49,908,620-49,908,119) of domestic dogs (Fig. 1). The five variant alleles between positions c. -21 and c. -46 were located within the microsatellite sequence on the $5^{\prime}$ untranslated region (5'UTR) in exon 1.

All variants were novel, with the exception of the commonly occurring $-46 \mathrm{~T}>\mathrm{C},-228 \mathrm{C}>\mathrm{A}$, and $-350 \mathrm{C}>\mathrm{A}$. These three single nucleotide variations were found in $35-50 \%$ of dogs. The previously unreported $-21 \mathrm{G},-27 \mathrm{~A}$, and $-43 \mathrm{~T}$ alleles were present in $14-36 \%$ of dogs (Table 1).

Several polymorphisms appear to be unique or much more frequent in dogs, when compared to their closest relatives, wolves and coyotes. For example, the c.-185 delT variant was found in $8 \%$ of dogs but not in the other Canis species (Table 1). MIDAS analysis also indicated that this base deletion appears to be the most recently evolved polymorphism in the $\operatorname{dog}$ GSTP1 promoter region. The c.-228 C > A polymorphism, present in almost half the dogs genotyped, was found in only $11 \%$ of wolves. At c. -46 , the least frequent variant in dogs $(\mathrm{C})$ is the major allele in wolves and coyotes. Similarly, the c.-305 GCCCTC repeat genotype differed between the three groups; in wolves and coyotes, the minor allele is the $(\mathrm{GCCCTC})_{2}$ repeat as opposed to

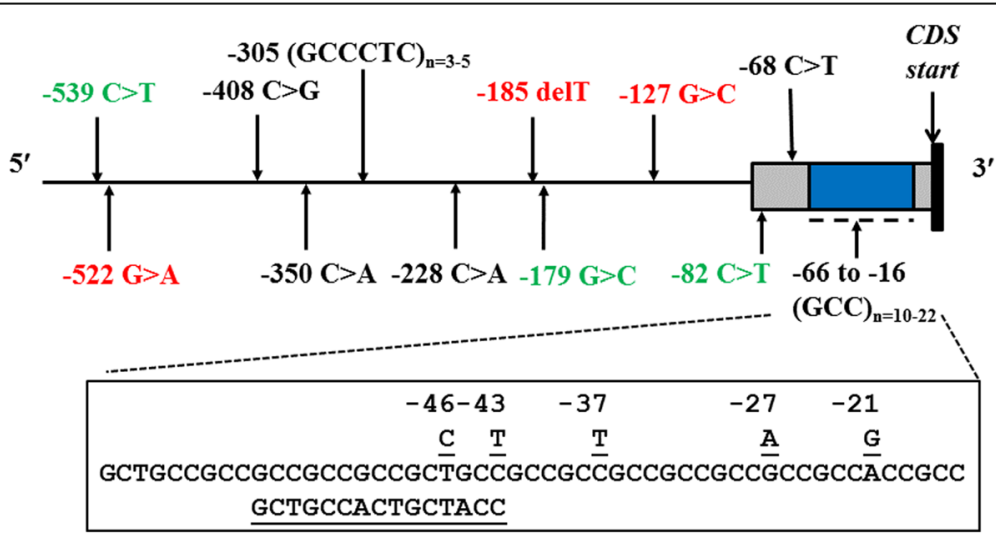

Fig. 1 Gene map displaying the position of GSTP1 promoter polymorphisms in the domestic dog and other canids. Exon 1 is shown as a shaded box, with its 3 'end representing the start of the coding sequence, which consists of a single adenine base. Variants found only in dogs are shown in red, while those exclusive to wolves and/or coyotes are in green. Inset shows the DNA sequence for the major microsatellite 17-repeat unit allele and the genetic polymorphisms found within this repeat region; the sequence of the compound repeat associated with certain alleles is shown below this sequence 
Table 1 GSTP1 promoter polymorphisms and minor allele frequencies (MAF) in domestic dogs, wolves and coyotes

\begin{tabular}{|c|c|c|c|c|c|}
\hline \multirow{2}{*}{$\begin{array}{l}\text { Polymorphism } \\
\text { accession no. }\end{array}$} & \multirow{2}{*}{$\begin{array}{l}\text { Position on dog } \\
\text { chromosome } 18\end{array}$} & \multirow[t]{2}{*}{ Sequence variant } & \multicolumn{3}{|l|}{ MAF } \\
\hline & & & Dog & Wolf & Coyote \\
\hline ss2019497428 & $49,908,119$ & c. $-21 A>G$ & 0.356 & 0.143 & 0.000 \\
\hline ss2019497429 & $49,908,125$ & c. $-27 \mathrm{G}>\mathrm{A}$ & 0.257 & 0.257 & 0.429 \\
\hline ss3021042895 & $49,908,135$ & c. $-37 \mathrm{C}>\mathrm{T}$ & 0.002 & 0.000 & 0.286 \\
\hline ss3021042898 & $49,908,141$ & c. $-43 \mathrm{C}>\mathrm{T}$ & 0.138 & 0.036 & 0.429 \\
\hline rs22633673 & $49,908,144$ & c. $-46 \mathrm{~T}>\mathrm{C}$ & 0.496 & 0.857 & 0.643 \\
\hline \multirow[t]{2}{*}{ ss2019497431 } & $49,908,166$ & c. $-68 \mathrm{C}>\mathrm{T}$ & 0.131 & 0.036 & 0.214 \\
\hline & $49,908,180$ & c. $-82 \mathrm{C}>\mathrm{T}$ & 0.000 & 0.143 & 0.143 \\
\hline \multirow[t]{2}{*}{ ss2019497432 } & $49,908,226$ & C. $-127 \mathrm{G}>\mathrm{C}$ & 0.009 & 0.000 & 0.000 \\
\hline & $49,908,277$ & c. $-179 \mathrm{G}>\mathrm{C}$ & 0.000 & 0.143 & 0.071 \\
\hline ss2019497433 & $49,908,283$ & c.-185 delT & 0.079 & 0.000 & 0.000 \\
\hline rs22646524 & $49,908,326$ & c. $-228 \mathrm{C}>\mathrm{A}$ & 0.464 & 0.107 & 0.143 \\
\hline \multirow[t]{3}{*}{ ss2019497435-6 } & $49,908,403$ & c. $-305(\mathrm{GCCCTC})_{2}$ & 0.000 & 0.143 & 0.214 \\
\hline & & c. $-305(\mathrm{GCCCTC})_{3}$ & 0.049 & 0.036 & 0.000 \\
\hline & & C.-305(GCCCTC $)_{5}$ & 0.002 & 0.000 & 0.000 \\
\hline rs22646522 & $49,908,448$ & c. $-350 \mathrm{C}>\mathrm{A}$ & 0.379 & 0.107 & 0.286 \\
\hline ss2019497438 & $49,908,506$ & c. -408 C > G & 0.013 & 0.000 & 0.286 \\
\hline \multirow[t]{2}{*}{ ss2019497439 } & $49,908,620$ & c. $-522 \mathrm{G}>\mathrm{A}$ & 0.005 & 0.000 & 0.000 \\
\hline & $49,908,637$ & c. -539 C > T & 0.000 & 0.036 & 0.286 \\
\hline
\end{tabular}

${ }^{\mathrm{a}}$ With respect to dog genome

the $(\mathrm{GCCCTC})_{3}$ variant in dogs. A total of 4 variants were only observed in wolves and coyotes.

The 5'UTR microsatellite, located 16 residues upstream of the start codon, was highly polymorphic (Table 2). This tandem repeat consists of a GCT followed by a series of (GCC) triplet units of variable length, with purities ranging from $80.3 \%(22 * 1$ or $22 * 2$ alleles) to $97.9 \%(16 * 1$ allele). In dogs, the most common repeats were 16 and 17 , occurring in $86 \%$ of the study population. The prevalent microsatellite length in the other canids was 18 in wolves, and 10 and 12 in coyotes. The 11-repeat unit motif, seen in $10 \%$ of dogs, was not found in their wild relatives.

Table 2 5' UTR microsatellite repeat length diversity, sequence and occurrence observed in dog (D), wolf (W), and coyote (C)

\begin{tabular}{|c|c|c|c|c|c|c|}
\hline Repeat unit & Sequence & VAR score & $\mathrm{dG}(\mathrm{kcal} / \mathrm{mol})$ & D & W & C \\
\hline 10 & $\mathrm{GCT}(\mathrm{GCC})_{7} \mathrm{ACCGCC}$ & 0.58 & -5.6 & 0.007 & 0.000 & 0.286 \\
\hline 11 & $\mathrm{GCT}(\mathrm{GCC})_{8} \mathrm{ACCGCC}$ & 0.75 & -8.5 & 0.099 & 0.000 & 0.000 \\
\hline $12^{*} 1$ & $\mathrm{GCT}(\mathrm{GCC})_{9} \mathrm{ACCGCC}$ & 0.88 & -9.5 & 0.000 & 0.040 & 0.000 \\
\hline $12 * 2$ & $\mathrm{GCT}(\mathrm{GCC})_{3} \mathrm{GCTGCCACTGCTACCGCCACCGCC}$ & 0.72 & -4.8 & 0.000 & 0.000 & 0.286 \\
\hline 13 & $\mathrm{GCT}(\mathrm{GCC})_{10} \mathrm{ACCGCC}$ & 0.97 & -11.5 & 0.000 & 0.180 & 0.000 \\
\hline 14 & $\mathrm{GCT}(\mathrm{GCC})_{13}$ & 1.09 & -14.5 & 0.013 & 0.000 & 0.000 \\
\hline 15 & $\mathrm{GCT}(\mathrm{GCC})_{14}$ & 1.12 & -17.5 & 0.005 & 0.000 & 0.000 \\
\hline $16^{*} 1$ & $\mathrm{GCT}(\mathrm{GCC})_{15}$ & 1.13 & -17.5 & 0.302 & 0.140 & 0.000 \\
\hline $16^{*} 2$ & $\mathrm{GCT}(\mathrm{GCC})_{3} \mathrm{GCTGCCACTGCTACC}(\mathrm{GCC})_{3} \mathrm{ACCGCCACCGCC}$ & 0.58 & -7.3 & 0.124 & 0.000 & 0.143 \\
\hline 17 & $\mathrm{GCT}(\mathrm{GCC})_{5} \mathrm{GCT}(\mathrm{GCC})_{8} \mathrm{ACCGCC}$ & 1.10 & -14.4 & 0.424 & 0.214 & 0.143 \\
\hline 18 & $\mathrm{GCT}(\mathrm{GCC})_{5} \mathrm{GCT}(\mathrm{GCC})_{9} \mathrm{ACCGCC}$ & 1.10 & -18.0 & 0.009 & 0.285 & 0.000 \\
\hline 19 & $\mathrm{GCT}(\mathrm{GCC})_{5} \mathrm{GCT}(\mathrm{GCC})_{6} \mathrm{ACCGCCACCGCCACCGCC}$ & 1.07 & -14.4 & 0.013 & 0.000 & 0.000 \\
\hline 20 & $\mathrm{GCT}(\mathrm{GCC})_{3} \mathrm{GCTGCCACTGCTACC}(\mathrm{GCC})_{3} \mathrm{ACC}(\mathrm{GCC})_{3} \mathrm{ACCGCCACCGCC}$ & - & -11.4 & 0.000 & 0.140 & 0.143 \\
\hline $22^{*} 1$ & $\mathrm{GCT}(\mathrm{GCC})_{3} \mathrm{GCTGCAACTGCTACC}(\mathrm{GCC})_{3} \mathrm{ACTGCTACC}(\mathrm{GCC})_{3} \mathrm{ACCGCCACCGCC}$ & 0.64 & -11.0 & 0.002 & 0.000 & 0.000 \\
\hline $22^{*} 2$ & $\mathrm{GCT}(\mathrm{GCC})_{3} \mathrm{GCTGCAACTGCTACC}(\mathrm{GCC})_{2} \mathrm{ACTGCTACC}(\mathrm{GCC})_{4} \mathrm{ACCGCCACCGCC}$ & - & -13.3 & 0.002 & 0.000 & 0.000 \\
\hline
\end{tabular}

Sequences shown are $5^{\prime} \rightarrow 3^{\prime}$ on the (-) strand. The SERV VARScore refers to the consensus GCC sequence. Computed Gibbs' energy contributions (dG) shown are for the most stable RNA secondary structure motif only 
Moreover, several repeat unit lengths appear to be unique to wolves $(12,13,20)$, and coyotes $(12,20)$. Both simple and compound repeats were observed for alleles with 16 and 22 repeat units. For example, a $(\mathrm{GCC})_{5}$ stretch (from -54 to -37$)$ in the 16 repeat allele is replaced by GCTGCCACTGCTACC. The compound 16-repeat allele containing this sequence, referred to as $16^{*} 2$, was observed in a third of dogs (or $12.4 \%$ of the entire study population) that had the 16 simple triplet repeat microsatellite allele (denoted as 16*1). The longest 22-repeat allele, which was observed in the heterozygous state in only two dogs, also contains the sequences GCTGCAACTGCTA $\mathrm{CC}(\mathrm{GCC})_{2}$ or $3 \mathrm{ACTGCTACC}(\mathrm{GCC})_{1}$ or 2 (denoted as $22 \% 1$ and $22 \% 2$ respectively). The $12 \% 2$ - and 20-repeat alleles, observed in wolves and coyotes, contained a GCTGC CACTGCTACC motif that is identical to the one observed in the $16^{*} 2$ allele in dogs. This motif was very common in coyotes, being observed in 5 out of the 7 coyotes (3 homozygotes; 2 heterozygotes) that were genotyped.

The 5'UTR tandem repeat was predicted to form a hairpin RNA structure at the $5^{\prime}$ end of the GSTP1 transcript. The stability of this secondary structure, as indicated by the Gibbs' energy (dG), decreases with smaller numbers of tandem repeats. Comparison of alleles of equal length but differing purity (for example $12 * 1$ vs $12 \% 2$, and $16 * 1$ vs $16 * 2$ ) showed that the compound repeat alleles were expected to be half as stable as their simple repeat counterparts, perhaps because the $5^{\prime}$ hairpin loop is missing or is structurally altered (Additional file 2a). This decreased stability appears to extend to the entire GSTP1 transcript (Additional file 2b). The VARScores indicate that the highest mutation rates, after consideration of the number of GCC repeat units, unit length and repeat purity should occur in the commonly occurring $16^{* 1} 1$ and 17 alleles in dogs and the 18 allele in wolves (Table 2). Conversely, the repeats least likely to experience GCC deletions or insertions are the smallest-length 10 repeat and the compound $16 * 2$ and $22 * 1$ repeats.

The gray fox GSTP1 promoter sequence was more similar to dog (98.2\%), than to red fox (96.1\%), which in turn showed more similarity to gray fox $(96.1 \%)$ than $\operatorname{dog}(95.9 \%)$. Alignment of the promoter sequence of red and gray fox with dog showed differences at 16 and 12 loci respectively, with four of these loci shared between the two fox species (Additional file 3). With one exception (the $5^{\prime}$ UTR microsatellite), none of these were located at the sites of the genetic polymorphisms observed in the Canis species. In the grey fox, there were two repeating GCCCTC units at the locus which corresponds to c.-305 in the dog; this repeat number was only observed in three wolves and two coyotes but in none of the domestic dogs. In the red fox, two of the four repeating units were changed to GCCCCCGCCCCC. In the 7 red foxes sequenced, no polymorphisms were observed within the entire promoter region, including the tandem repeat. The red foxes were all homozygous for a 12-repeat exon 1 microsatellite $\left(\mathrm{GCC}(\mathrm{GCC})_{3} \mathrm{ACC}(\mathrm{GCC})_{3}\right.$ (ACCG $\mathrm{CC})_{2}$ ) that was distinct from the equal-length alleles observed in wolves $(12 * 1)$ and coyotes (12*3) (Table 2). The single gray fox sequenced had a unique 8-repeat/16-repeat microsatellite genotype (GCT(GCC) $)_{7} \mathrm{ACCGCC}$ and GCT $(\mathrm{GCC})_{3}$ GCTGCCACTGCTGGCT(GCC) ${ }_{5}$ ACCGCC), with a single base (underlined) interrupting the triplet repeat sequence.

Alignment of the 'wild-type' GSTP1 transcript (which includes the 17-repeat allele) with other mammalian transcripts showed evidence of the microsatellite in a number of other species, with the highest number of repeats (13) observed in the white-tailed deer and Bactrian camel. The other species all showed repeat numbers lower than 10, with the two rodent species not showing any GCC repeat units in their 5'UTR (Additional file 4). No information is available on whether these sequences are polymorphic, as has been observed in the three canid species described in this study.

Using the genotypes from homozygous dogs, 28 distinct GSTP1 promoter haplotypes could be constructed in the dog (Additional file 5). Fifteen of these haplotypes were observed only in the heterozygous state and thus could only be inferred. An additional 8 haplotypes were only found in wolves and/or coyotes. Designation of haplotypes was based on the nucleotide sequence of the reference boxer genome assembly (Canfam 3.0), with the wild-type or most common sequence designated as *1A. Haplotype names were first numbered according to the genetic polymorphism pattern in the promoter region upstream of the microsatellite region (that is, upstream from c.-68). Each haplotype was then designated with letters after the number, each letter corresponding to a specific SNP pattern within the microsatellite. Each haplotype name ends with a number in parentheses, which refers to the repeat unit length found associated with each specific allele (Table 3 ). The common *1A, *2A, and "6A haplotypes were associated with two or more different tandem repeat units (Additional file 5). A similar phenomenon was observed for the "1H haplotype in wolves, with four different repeat lengths (18 and 13-unit predominating) observed occurring with this allelic arrangement. While dogs share five haplotypes with wolves and one with coyotes, there are significant differences. The commonest haplotypes in dogs are ${ }^{*} 1 \mathrm{~A}(17)$ and ${ }^{*} 6 \mathrm{~A}(16)$ (accounting for almost half of all dogs), while the prevalent alleles in wolf and coyote are, respectively, ${ }^{*} 1 \mathrm{H}(17)$ and ${ }^{*} 1 \mathrm{C}(10)$, which were either absent or occurring at low frequency in the dogs. The *3A(11) haplotype, characterized by the $\mathrm{T}$ deletion at -185 and a microsatellite length of 11 repeats, was observed in around $10 \%$ of dogs but was not observed in coyotes and 
Table 3 Comparison of GSTP1 promoter haplotype in dogs, wolves and coyotes

\begin{tabular}{llll}
\hline Haplotype & Dog & Wolf & Coyote \\
\hline *1A (17) & 0.257 & 0.179 & 0.071 \\
*1C (10) & 0.007 & 0.000 & 0.286 \\
*1H (17) & 0.000 & 0.286 & 0.000 \\
*1H (13) & 0.000 & 0.143 & 0.000 \\
*2A (16) & 0.077 & 0.000 & 0.000 \\
*3A (11) & 0.081 & 0.071 & 0.000 \\
*6A (16) & 0.218 & 0.036 & 0.000 \\
*10A (16) & 0.040 & 0.000 & 0.000 \\
*11A (16) & 0.09 & 0.071 & 0.143 \\
*17A(20) & 0.000 & 0.036 & 0.071 \\
*19A (20) & 0.000 & 0.000 & 0.071 \\
*20A (17) & 0.000 & 0.000 & 0.071 \\
*21A (12) & 0.000 & 0.000 & 0.214 \\
*22A (12) & 0.000 & 0.000 & 0.071 \\
$\hat{\text { H }}$ & $\mathbf{0 . 8 5 9}$ & $\mathbf{0 . 8 7 8}$ & $\mathbf{0 . 9 0 2}$
\end{tabular}

$\hat{\mathrm{H}}$ : Haplotype diversity (in bold); only haplotype frequencies $>0.05$ shown. Number in parentheses denotes $5^{\prime}$ UTR microsatellite repeat length

wolves. Haplotypes with microsatellite unit lengths greater than 17, while common in wolves and coyotes (haplotype frequencies of 0.393 and 0.142 respectively), were uncommon in dogs (haplotype frequency: 0.029).

Promoter haplotypes were compared for breeds in which at least five dogs or more were represented (Table 4). A wide inter-breed variation in the type and extent of genetic diversity was observed. Chihuahuas and Beagles had the highest haplotypic diversity (a measure of the uniqueness of a particular haplotype in a given population). On the other hand, 20 out of 22 Boxers, and all the Siberian Huskies, had a $" 1 \mathrm{~A}(17) / * 1 \mathrm{~A}(17)$ diplotype, resulting in a very low or no haplotypic diversity. The commonest haplotype in dogs, " $1 \mathrm{~A}(17)$, was not found in Basenjis and Dobermans. The $-408 \mathrm{C}>\mathrm{G}$ transversion occurring in 6 out of 11 Standard Poodles (found only in the "8A(19) haplotype), was, with the exception of one Shar-Pei absent in all other dogs, but, interestingly was also found in 3 out of 7 coyotes ("21A and "21B haplotypes). The *3A(11) haplotype was over-represented in older breeds such as Afghan Hounds, Basenjis, and Greyhounds. Haplotypes with the $16 * 2$ compound repeat ("9A, "10A) occurred more often in more modern breeds such as Beagles, Dobermans, and Golden Retrievers.

Some uncommon haplotypes, such as ${ }^{*} 1 \mathrm{C}(10)$, were detected in one or two breeds only ( 3 alleles in two Chow Chows; 1 allele in a Tibetan terrier). Interestingly, this haplotype was also observed in 6 coyotes. The otherwise rare "6A(17) haplotype was seen 5 times in the three New Guinea Singing Dogs investigated, with the other Singing Dog having the commoner "6A(16) haplotype.

The mechanisms by which these polymorphisms could affect GSTP1 expression via effects on transcription factor binding were investigated using the web-based program LASAGNA-Search. The wolf and coyote $-539 \mathrm{C}>\mathrm{A}$ SNP may delete the binding sites for ARE86 (TRANSFAC Accession number M00415), Pax-2 (M00486), and C/EBP (M00159) and introduce novel sites for interactions with CHOP:C/EBP alpha (M00249) and STAT4 (M000498), while the 408G variant deletes an ARP-1 (M00155) site and creates a new site for Brachyury protein (M00150)

Table 4 Comparison of GSTP1 promoter haplotype frequencies between different dog breeds

\begin{tabular}{|c|c|c|c|c|c|c|c|c|c|c|c|c|c|c|}
\hline Haplotype & All dogs & Afg & Bas & Sib & Chi & Gre & Box & Lab & Pem & Bea & Dob & $\mathrm{Gol}$ & Ger & Poo \\
\hline${ }^{*} 1 \mathrm{~A}(17)$ & 0.257 & 0.200 & 0.000 & 1.000 & 0.300 & 0.071 & 0.932 & 0.125 & 0.100 & 0.115 & 0.000 & 0.300 & 0.545 & 0.045 \\
\hline${ }^{*} 1 \mathrm{~A}(18)$ & 0.004 & 0.000 & 0.200 & 0.000 & 0.000 & 0.000 & 0.000 & 0.000 & 0.000 & 0.000 & 0.000 & 0.000 & 0.000 & 0.000 \\
\hline${ }^{*} 1 \mathrm{~B}(17)$ & 0.086 & 0.000 & 0.000 & 0.000 & 0.000 & 0.286 & 0.023 & 0.125 & 0.000 & 0.231 & 0.000 & 0.000 & 0.091 & 0.045 \\
\hline${ }^{*} 2 A(16)$ & 0.077 & 0.000 & 0.000 & 0.000 & 0.200 & 0.071 & 0.000 & 0.063 & 0.100 & 0.038 & 0.333 & 0.200 & 0.000 & 0.318 \\
\hline${ }^{*} 2 \mathrm{~B}(17)$ & 0.013 & 0.000 & 0.000 & 0.000 & 0.100 & 0.000 & 0.000 & 0.000 & 0.000 & 0.000 & 0.000 & 0.000 & 0.045 & 0.000 \\
\hline${ }^{*} 3 \mathrm{~A}(11)$ & 0.081 & 0.600 & 0.500 & 0.000 & 0.000 & 0.571 & 0.023 & 0.125 & 0.100 & 0.000 & 0.000 & 0.000 & 0.000 & 0.000 \\
\hline${ }^{*} 5 \mathrm{~A}(17)$ & 0.011 & 0.000 & 0.000 & 0.000 & 0.100 & 0.000 & 0.000 & 0.000 & 0.000 & 0.077 & 0.000 & 0.000 & 0.000 & 0.000 \\
\hline${ }^{*} 6 \mathrm{~A}(16)$ & 0.218 & 0.100 & 0.100 & 0.000 & 0.100 & 0.000 & 0.000 & 0.438 & 0.300 & 0.269 & 0.417 & 0.050 & 0.182 & 0.227 \\
\hline${ }^{*} 6 \mathrm{~A}(17)$ & 0.027 & 0.000 & 0.000 & 0.000 & 0.000 & 0.000 & 0.000 & 0.000 & 0.000 & 0.000 & 0.000 & 0.000 & 0.136 & 0.000 \\
\hline *7A(16) & 0.005 & 0.000 & 0.000 & 0.000 & 0.000 & 0.000 & 0.000 & 0.000 & 0.200 & 0.000 & 0.000 & 0.000 & 0.000 & 0.000 \\
\hline *8A(19) & 0.013 & 0.000 & 0.000 & 0.000 & 0.000 & 0.000 & 0.000 & 0.000 & 0.000 & 0.000 & 0.000 & 0.000 & 0.000 & 0.318 \\
\hline${ }^{*} 9 A(16)$ & 0.072 & 0.100 & 0.000 & 0.000 & 0.100 & 0.000 & 0.000 & 0.063 & 0.200 & 0.154 & 0.083 & 0.300 & 0.000 & 0.000 \\
\hline${ }^{*} 10 \mathrm{~A}(16)$ & 0.040 & 0.000 & 0.000 & 0.000 & 0.000 & 0.000 & 0.000 & 0.063 & 0.000 & 0.077 & 0.167 & 0.000 & 0.000 & 0.000 \\
\hline$\hat{\mathrm{H}}$ & 0.860 & 0.644 & 0.756 & 0.000 & 0.911 & 0.626 & 0.133 & 0.803 & 0.889 & 0.853 & 0.741 & 0.809 & 0.672 & 0.777 \\
\hline
\end{tabular}

Only those haplotypes with frequencies higher than 0.01 are shown. Afg Afghan Hound, Bas Basenji, Sib Siberian Husky, Chi Chihuahua, Gre Greyhound, Box Boxer, Lab Labrador Retriever, Pem Pembroke Welsh Corgi, Bea Beagle, Dob Doberman Pinscher, Gol Golden Retriever, Ger German Shepherd, Poo Standard Poodle. H. Haplotype diversity shown in bold 
binding. The common $350 \mathrm{C}>\mathrm{A}$ and $-228 \mathrm{C}>\mathrm{A}$ SNPs result in new sites for the binding of NRF-2 (M00108) and GATA (M00076, M00462, M00075) respectively. The rare $-127 \mathrm{C}$ allele is predicted to result in a new GC box (M00255) and E2 binding site (M00107, M00181). The wolf and coyote $-82 \mathrm{C}>\mathrm{T}$, located just inside the $5^{\prime} \mathrm{UTR}$, may generate a new PPARalpha:RXR-alpha (M00242) binding site, while the other 5'UTR SNP, $-68 \mathrm{C}>\mathrm{T}$, also unique to these wild canids, creates new sites for the binding of HNF-4alpha1 (M00411) and COUP-TF (M00158). The Wilms Tumor zinc finger (WT1-KTS) protein (T01839) was predicted to bind to the multiple EGR1 GGCG core sequences (on the + strand) within the microsatellite. Binding sites for this transcription factor were decreased for alleles with a smaller number of repeat units, and in repeats with decreased purity. For example, while there were 25 potential binding sites on the $16^{*} 1$-repeat allele, there were only 11 sites on the 11-repeat allele, and just 5 sites on the similar size but compound 16*2-repeat allele (Additional file 6). On the other hand, alleles with the GCTGCCACTGCTACC motif $(12 * 2,16 * 2,20,22$ repeats) were predicted to have a new binding site for KLF12 (M00468). In the case of the "22-repeat alleles, the unique GCA triplet within the repeat introduces a binding site for MyoD (M00184) that binds to the consensus TGCAACTGCT.

\section{Discussion}

The promoter region of a gene is essential for its proper function, containing enhancers and repressors that control its rate of expression. Certain promoter features, such a TATA box, cis mutations, and the presence of unstable tandem repeats, increase expression divergence, or the capacity of a gene to evolve in expression [37]. Genes containing these type of promoters preferentially diverge when the effects of natural selection are minimized [38], a good example of which is canine domestication. The DNA resequencing performed in this study demonstrated that the canine GSTP1 promoter region (with a TAATAA box located at c.-102) contains a number of genetic polymorphisms of varying frequency and a highly polymorphic tandem repeat sequence located in the $5^{\prime}$ untranslated region. It is possible that the promoter architecture found in the canine GSTP1 gene has arisen both to allow flexibility in the biotransformation of existing and new xenobiotics as well as a direct consequence of inbreeding practices.

Molecular phylogeny studies have shown that the dog family, or Canidae, can be classified into four clades, including the wolf-like canids (dog, gray wolf, coyote), the fox-like canids (red fox), the South American canids, and the smallest, most primitive clade represented by the grey fox and the island fox [39]. The dog GSTP1 promoter sequence showed the best similarity with the other Canis species, gray wolf and coyote, followed by the gray fox, and red fox. Although sample sizes for the wild canids used in our study were too small for definite conclusions, we can tentatively conclude that our data supports the aforementioned phylogeny studies.

A total of 16 polymorphic loci were identified in the canid GSTP1 promoter, of which 13 were polymorphic in the dog population, 11 were polymorphic in the wolf, and 12 were polymorphic in the coyote. Almost half the dogs sequenced had the wild-type "1A(17) and the "6A(16) haplotype. Three loci (c.-82, c.-179, c.-539) were seen to have the allele fixed in dogs but polymorphic in both wolves and coyotes, which likely reflects the bottlenecks associated with domestication or breed formation. The c.$46 \mathrm{C}>\mathrm{T}$ allele was observed in $50 \%$ of dogs but only in $14 \%$ of wolves and $36 \%$ of coyotes, suggesting that this polymorphism may have arisen from domestication, and that the ancestral allele for this SNP (rs22633673) should therefore be ' $\mathrm{C}$ '. Nine different microsatellite repeat lengths were observed in dogs, while six and five repeat lengths were seen in wolves and coyotes respectively. The *3A(11) dog haplotype is characterized by the 11-repeat microsatellite, which is in strong linkage disequilibrium with the deletion variant at c.-185. This haplotype was not observed in wolves and coyotes and appears to have arisen as a consequence of dog domestication. The "3A(11) haplotype may thus prove useful in DNA marker studies seeking to distinguish between domestic dogs and their wild relatives. Another potential marker is the $1 \mathrm{H}(\mathrm{n})$ haplotype, which is found at high frequency in wolves but is absent in both dogs and coyotes. Haplotypes which are unique to and found in almost half of the coyotes studied were the "1C (10) and the "21A (12) haplotypes. Interestingly, the *1C (10) was also found in 2 Chow Chows and 1 Tibetan Terrier, both of which are ancient breeds [40] and would conceivably retain more ancestral alleles than the more recent breed groups.

The fox species were not polymorphic at any of the loci common to the Canis species, and exhibited sequence dissimilarities at 21 different positions. The lack of variation between the 7 foxes sequenced, including those polymorphisms within the microsatellite region, was surprising in view of the fact that 4 red foxes originated from different geographical areas in the US and the other 3 were Russian domesticated foxes. It would be interesting to further investigate microsatellite variation in a greater number of coyotes, wolves and foxes, as well as other Canis and Canidae species.

Since most dog breeds represent closed breeding populations that receive limited genetic variation [41], we expected to find significant differences between dog breeds, as well as a lack of variation within these breeds. The "3A (11) haplotype predominated in Afghans, Basenjis and Greyhounds, while the commonest haplotypes in Standard Poodles were *2A(16) and "8A(19), the 
latter haplotype occurring only in this breed. A lack of genetic diversity in the GSTP1 promoter was observed in Boxers and Siberian Huskies. Boxers are known to have low levels of heterozygosity, this being one of the reasons why a female dog from this breed was selected to have its genome sequenced at the Broad Institute [42]. Despite the fact that the 6 Siberian Huskies in the study were not related and came from different states within the US, they had identical "1A(17) haplotypes. This lack of genetic diversity is surprising in view of the fact that Siberian Huskies have ancient origins and display high heterozygosity in microsatellite data compared with other breeds [40].

The canine GSTP1 promoter containsa microsatellite mainly consisting of GCC triplet units tandemly repeated anywhere from 10 to 23 times. This repeat exhibits a very diverse distribution across species, even across closely related species, indicating rapid evolutionary change. Significant expansion of this microsatellite may have occurred in species belonging to the genus Canis, as it appears that the corresponding region in other mammals is much shorter. The red fox sequences were not polymorphic, perhaps indicating that the longer repeats occurred with the appearance of extant canids around 10 million years ago. Similarly, a GCC dodecamer repeat found in the coding region of the canine EPM2 gene has been shown to be polymorphic across the Canidae but not to other close relatives of the $\operatorname{dog}$ [43].

The microsatellite in the canine GSTP1 promoter has a very high GC content, which means that there is a greater tendency to form hairpin-loop and slipped-strand DNA structures during replication, recombination, and repair. These structures result in genetic instability and increased polymorphisms [44]. Two mechanisms are probably the reason for the microsatellite polymorphisms observed. One is replication slippage, which occurs when the daughter strand slips back one or more repeating unit in pairing with the template strand, leading to a trinucleotide expansion or contraction during DNA replication. This probably accounts for alleles which only differ by one repeat unit, as in the 14, 15 and $16 " 1$ repeats seen in the " $2 \mathrm{~A}$ and " $6 \mathrm{~A}$ haplotypes, and possibly even those alleles that differ by two GCC units (for example, 14 and 16*1). The other mechanism is unequal recombination or crossing-over, which is a type of gene duplication or deletion event that deletes a sequence in one strand and replaces it with a duplication from its sister chromatid in mitosis or from its homologous chromosome during meiosis. Based on the microsatellite alleles observed, several types of crossover events can be postulated to occur via intra-repeat and/or inter-repeat recombination mechanisms. The simplest and most common event may involve the exchange of one GCC repeat at multiple sites, generating, for example, the 15 and 17 alleles from two parent $16^{*} 1$ alleles (Fig. 2a). Identical-length alleles may also recombine only at specific sites on the tandem repeat, as may occur when two $16 * 2$ alleles exchange 4 repeats to form the compound $12 * 2$ and 20 alleles, as was observed in coyotes (Fig. 2b). Unequal exchanges of repeats can occur between alleles with different lengths, as between the 17 and $16^{*} 1$ alleles to form daughter alleles with 11 and 22 repeats (Fig. 2c), or alleles with 15 and 18 repeats. Whilst the longer allele was observed in only two dogs, the truncated 11 allele was the third most frequent allele observed, probably because the 16- and 17-repeat unit alleles are the most common in the dog. The 19-repeat unit allele seen in standard poodles could be another example of unequal crossing-over between 2 different alleles, in this case the 17 and 18 repeat alleles, which also generates the common $16^{*} 1$ allele (Fig. 2d). Support for this mechanism comes from the fact that all the standard poodles that were heterozygous for the 19 allele had the $16^{*} 1$ allele. Moreover, the $16^{*} 1$ and 19 alleles represent the major forms of the microsatellite in the standard poodles sequenced, with allele frequencies
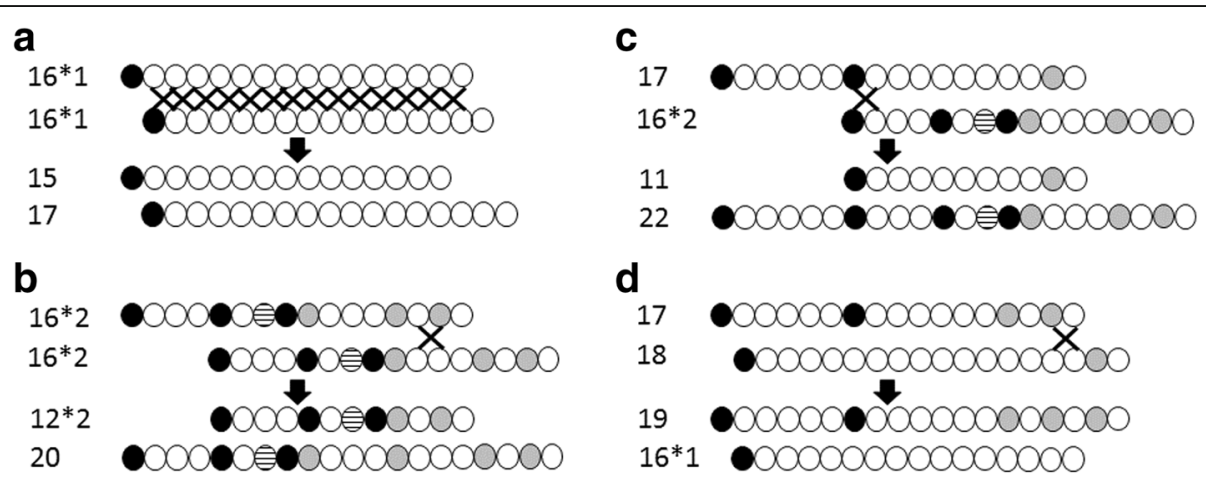

$\bigcirc$ GCC $\bigcirc \mathrm{GCT} \bigcirc \mathrm{ACC} \ominus \mathrm{ACT}$

Fig. 2 Derivation of GSTP1 5'UTR microsatellite alleles by unequal recombination between identical $(\mathbf{a}, \mathbf{b})$ and distinct (c, $\mathbf{d})$ repeat sequences. Possible point of an exchange is indicated by an ' $X$ ' 
of 0.59 and 0.32 respectively. Finally, an ancestral recombination event could account for the origin of the common $\operatorname{dog} 16^{*} 2$ and 17 alleles from crossing-over the 13- and 20-repeat variants that appear to be found only in wolves.

In humans, it has been estimated that a third of promoter variants may alter gene expression to a functionally significant extent [45]. Several studies have demonstrated that tandem GGC or CCG repeats in gene promoters have significant effects on gene function. For example, expansion of a CGG repeat in the 5'UTR of the fragile site mental retardation 1 (FMR1) gene causes epigenetic changes that reduce transcription factor binding and are the cause of Fragile X Syndrome [46, 47]. Two-repeat differences in GCC repeat length in a microsatellite located in the thiopurine S-methyltransferase (TPMT) 5'UTR may be responsible for ultra-high enzyme activity and consequent resistance to treatment by thiopurine drugs [48]. Tandem repeats can modulate GSTP1 expression by changing the copy number of binding sites for transcription factors, as has been observed with the binding of Sp1 to the human $E G F$ gene [49], the interactions of Sp1 and AP2 to the human RFC gene [50], and the binding of p53 to PIG3 [51]. The GSTP1 microsatellite GCC repeat polymorphisms were predicted to affect the number of potential binding sites for the Wilms Tumor zinc finger (WT1-KTS) protein. The Wilms tumor gene 1 (WT1) is a transcription factor that acts as an oncogene in acute myeloid leukemia [52], and a recent Chip-Seq investigation has revealed that binding of the WT1-KTS isoform in leukemic cells is correlated to active transcription of numerous genes [53]. The $16 * 2$ allele, which was found in $12 \%$ of the dogs in the study and over-represented in breeds such as Pembroke Welsh Corgi and Golden Retrievers, has very few binding sites for the WT1 activator compared to the other major repeat alleles (11, 16*1 and 17). The CAGTGGc motif present in the $16^{*} 2$ allele provides a binding site for the WT-1 homolog transcriptional silencer KLF12 (Ap-2rep). It has been hypothesized that KLF-12 silences WT-1dependent gene expression [54]. The decreased binding of WT1 and novel binding of KLF12 to the repeat could decrease GSTP1 expression. Although there is as yet no experimental evidence of the binding of WT1 and/or KLF12 to the canine GSTP1 promoter, it would be interesting to establish whether these transcription factors do indeed bind to this repeat sequence and whether the polymorphisms observed affect the number of binding sites and, ultimately, gene expression.

The tandem repeat is located in the 5'UTR of the canine GSTP1 gene and therefore forms part of the mature mRNA. The microsatellite is also located in proximity to the core promoter (20-30 base pairs downstream of the predicted transcription start site and the TATA box respectively), so variations in the tandem repeats could interfere with the binding of RNA polymerase by changing the spacing between functional elements in promoters [55]. The microsatellite may also affect stability of the secondary structure of the GSTP1 transcript. In the dog, two types of microsatellites consisting of 16 triplet repeats were observed. The $16^{*} 1$ allele was predicted to form a more stable RNA hairpin-loop secondary structure than the $16 * 2$ allele. The $16 * 2$ allele alters the structure and position of the hairpin loop, potentially decreasing the stability of the entire GSTP1 transcript. These hairpin RNA structures may play a role in disease pathogenesis by affecting the processing of the primary transcript, resulting in deficit of the corresponding protein, or interacting with RNA-binding proteins, altering their normal activity [56].

\section{Conclusions}

In summary, this study has demonstrated the existence of multiple polymorphisms in the proximal promoter of the canid GSTP1 gene. Distinct haplotype differences exist between various dog breeds and between dogs, wolves, coyotes and foxes. The high degree of polymorphism in a 3'UTR microsatellite may be due to replication slippage and unequal allelic recombination events. Some of these variants may affect the binding of transcription factors and/or decrease mRNA stability, altering the detoxification potential of glutathione conjugation catalyzed by GSTP1. In order to investigate any effects that these promoter polymorphisms may have on the glutathione conjugation of xenobiotics, reporter gene assays are underway to determine the effect of the various microsatellite alleles on canine GSTP1 expression, together with the determination of GSTP1 mRNA levels and GSTP1 activity in the blood of genotyped dogs.

\section{Additional files}

Additional file 1: Details of individual dogs, wolves, coyotes, and foxes used in the study. (XLSX $20 \mathrm{~kb}$ )

Additional file 2: a RNA secondary structure of the GCC12*1, 12*2, $16^{*} 1$, and $16^{*} 2$ GSTP1 promoter 5'UTR repeat alleles. $\mathbf{b}$ Comparison of RNA secondary structure of canine GSTP1 transcripts with different 5'UTR repeat alleles. (ZIP $191 \mathrm{~kb}$ )

Additional file 3: Alignment of dog versus fox GSTP1 promoter sequences. (DOCX 26 kb)

Additional file 4: Alignment of the dog GSTP1 5'UTR with select mammalian GSTP1 transcripts. (DOCX $158 \mathrm{~kb}$ )

Additional file 5: Canid GSTP1 promoter haplotype and frequencies. (XLSX $16 \mathrm{~kb}$ )

Additional file 6: LASAGNA Search results. (XLSX $43 \mathrm{~kb}$ )

\section{Acknowledgements}

The authors would like to express their thanks to all the private dog owners that consented to have their dogs participate in this study, as well as the Des Moines Training \& Obedience Club, Paws \& Effect, Des Moines Kennel Club, Jordan Creek Animal Hospital, Ingersoll Animal Hospital, Wind Dancer Kennels. We also like to thank Denise Attard-Sacco, Sonya Loynahan, Amy 
Bassett, Heddie Leger, and Suzanne Popson for their help in canine recruitment efforts. Special thanks also to the following wild canid education centers for providing DNA samples from wolves, coyotes and foxes: Wolf Park, Colorado Wolf and Wildlife Center, JAB Canid Education and Conservation Center, and Blanke Park Zoo.

\section{Funding}

Drake University Research Grant 100,000-202 PRSAGX.

Drake College of Pharmacy and Health Science Harris Endowment 2016-17. SM supported by a 2016 Summer Drake Undergraduate Science Collaborative Institute Fellowship and a 2016-2017 lowa Space Grant Consortium Research Mentor Program Project number 103237. KT supported by a 2014 Summer Drake Undergraduate Science Collaborative Institute Fellowship.

\section{Availability of data and materials}

All polymorphism locus and population data was deposited in the short genetic variation database, dbSNP, at the National Institutes of Health website: http://www.ncbi.nlm.nih.gov/SNP/

\section{Authors' contributions}

JS designed the study and wrote the majority of the manuscript. SM and KT performed the experiments. JS and SM carried out the data analysis. All authors read and approved the final manuscript.

\section{Ethics approval and consent to participate}

The Drake IACUC reviewed the study protocol and the committee determined that these experiments did not require IACUC approval. The IACUC decision is reproduced below: -.

Date: February 9, 2017

From: Kimberly Huey, Ph.D. IACUC Chair.

To: James Sacco, Ph.D.

Re: Sequencing of the Glutathione-S-Transferase P-1 (GSTP1) gene promoter in canids.

The IACUC has reviewed your protocol "Sequencing of the Glutathione-STransferase P-1 (GSTP1) gene promoter in canids" and the committee has determined that these experiments do not require IACUC approval. This research is considered exempt from IACUC approval based upon the following stipulations:

1. Veterinarians or animal handlers will be collecting all the saliva samples and will provide these samples to your laboratory. No one in your laboratory will have any physical contact with the wolves.

2. No samples will be collected on the Drake University campus and no wolves will be housed in Drake University animal facilities.

If you subsequently plan to make any significant changes regarding the use of animals in this protocol you must inform the IACUC before continuing the research

Thank you for your cooperation and good luck with your research.

Kimberly Huey, PhD.

Associate Professor of Physiology.

Chair, IACUC.

Drake University.

271-4853.

\section{Consent for publication}

Not applicable.

\section{Competing interests}

The authors declare that they have no competing interests.

\section{Publisher's Note}

Springer Nature remains neutral with regard to jurisdictional claims in published maps and institutional affiliations.

Received: 19 July 2017 Accepted: 19 September 2017

Published online: 11 October 2017

\section{References}

1. Hayes JD, Flanagan JU, Jowsey IR. Glutathione transferases. Annu Rev Pharmacol Toxicol. 2005;45:51-88.

2. Sherratt PJ, Pulford DJ, Harrison DJ, Green T, Hayes JD. Evidence that human class Theta glutathione S-transferase T1-1 can catalyse the activation of dichloromethane, a liver and lung carcinogen in the mouse. Comparison of the tissue distribution of GST T1-1 with that of classes Alpha, Mu and Pi GST in human. Biochem J. 1997;326:837-46.

3. Bocedi A, Fabrini R, Lai O, Alfieri L, Roncoroni C, Noce A, Pedersen JZ, Ricci G. Erythrocyte glutathione transferase: a general probe for chemical contaminations in mammals. Cell Death Discov. 2016;2:e16029.

4. Berhane K, Widersten M, Engstrom A, Kozarich JW, Mannervik B. Detoxication of base propenals and other alpha, beta-unsaturated aldehyde products of radical reactions and lipid peroxidation by human glutathione transferases. Proc Natl Acad Sci U S A. 1994;91:1480-4.

5. Hu X, Xia H, Srivastava SK, Herzog C, Awasthi YC, Ji X, et al. Activity of four allelic forms of glutathione S-transferase hGSTP1-1 for diol epoxides of polycyclic aromatic hydrocarbons. Biochem Biophys Res Commun. 1997;238 397-402.

6. Abel EL, Opp SM, Verlinde CL, Bammler TK, Eaton DL. Characterization of atrazine biotransformation by human and murine glutathione S-transferases. Toxicol Sci. 2004:80:230-8.

7. Nagar R, Khan AR, Poonia A, Mishra PK, Singh S. Metabolism of cisplatin in the organs of Rattus norvegicus: role of Glutathione S-transferase P1. Eur J Drug Metab Pharmacokinet. 2015;40:45-51.

8. Parker LJ, Ciccone S, Italiano LC, Primavera A, Oakley AJ, Morton CJ, et al. The anti-cancer drug chlorambucil as a substrate for the human polymorphic enzyme glutathione transferase P1-1: kinetic properties and crystallographic characterisation of allelic variants. J Mol Biol. 2008;380:131-44.

9. Burim RV, Canalle R, Martinelli AL, Takahashi CS. Polymorphisms in glutathione S-transferases GSTM1, GSTT1 and GSTP1 and cytochromes P450 CYP2E1 and CYP1A1 and susceptibility to cirrhosis or pancreatitis in alcoholics. Mutagenesis. 2004;19:291-8.

10. Ntais C, Polycarpo A, loannidis JP. Association of GSTM1, GSTT1, and GSTP1 gene polymorphisms with the risk of prostate cancer: a meta-analysis. Cancer Epidemiol Biomark Prev. 2005;14:176-81.

11. Hu X, Herzog C, Zimniak P, Singh SV. Differential protection against benzo[a]pyrene-7,8-dihydrodiol-9,10-epoxide-induced DNA damage in HepG2 cells stably transfected with allelic variants of pi class human glutathione S-transferase. Cancer Res. 1999;59:2358-62.

12. Das P, Shaik AP, Bammidi VK. Meta-analysis study of glutathione-Stransferases (GSTM1, GSTP1, and GSTT1) gene polymorphisms and risk of acute myeloid leukemia. Leuk Lymphoma. 2009:50:1345-51.

13. Yu Z, Li Z, Cai B, Wang Z, Gan W, Chen H, et al. Association between the GSTP1 Ile105Val polymorphism and prostate cancer risk: a systematic review and meta-analysis. Tumour Biol. 2013;34:1855-63.

14. Chen X, Liang L, Hu X, Chen Y. Glutathione S-transferase P1 gene lle105Val polymorphism might be associated with lung cancer risk in the Chinese Han population. Tumour Biol. 2012;33:1973-81.

15. Wang Z, Xue L, Chong T, Li H, Chen H, Wang Z. Quantitative assessment of the association between glutathione S-transferase P1 lle105Val polymorphism and bladder cancer risk. Tumour Biol. 2013;34:1651-7.

16. Ma Y, Wei X, Han G, Xue M, Li G, Li Y. Glutathione S-transferase P1 Ile105Val polymorphism contributes to increased risk of gastric cancer in East Asians. Tumour Biol. 2013;34:1737-42.

17. Li W, Chen J, Liu C. Glutathione S-transferase P1 Ile105Val polymorphism and oral cancer risk: a meta-analysis. Int J Med Sci. 2013;10:392-8.

18. Sergentanis TN, Economopoulos KP. GSTT1 and GSTP1 polymorphisms and breast cancer risk: a meta-analysis. Breast Cancer Res Treat. 2010;121:195-202.

19. Kordi-Tamandani DM, Mojahed A, Sahranavard R, Najafi M. Association of glutathione s-transferase gene methylation with risk of schizophrenia in an Iranian population. Pharmacology. 2014;94:179-82.

20. Zhang R, Kang KA, Piao MJ, Kim KC, Zheng J, Yao CW, et al. Epigenetic alterations are involved in the overexpression of glutathione S-transferase $\pi$ 1 in human colorectal cancers. Int J Oncol. 2014;45:1275-83.

21. Challouf S, Ziadi S, Zaghdoudi R, Ksiaa F, Ben Gacem R, Trimeche M. Patterns of aberrant DNA hypermethylation in nasopharyngeal carcinoma in Tunisian patients. Clin Chim Acta. 2012;413:795-802.

22. Lasabova Z, Tilandyova P, Kajo K, Zubor P, Burjanivova T, Danko J, et al. Hypermethylation of the GSTP1 promoter region in breast cancer is associated with prognostic clinicopathological parameters. Neoplasma. 2010;57:35-40.

23. Hayes HM, Tarone RE, Cantor KP, Jessen CR, McCurnin DM, Richardson RC. Case-control study of canine malignant lymphoma: positive association with dog owner's use of 2,4-dichlorophenoxyacetic acid herbicides. J Natl Cancer Inst. 1991;83:1226-31. 
24. Hayes HM, Tarone RE, Cantor KP. On the association between canine malignant lymphoma and opportunity for exposure to 2,4dichlorophenoxyacetic acid. Environ Res. 1995;70:119-25.

25. Takashima-Uebelhoer BB, Barber LG, Zagarins SE, Procter-Gray E, Gollenberg AL, Moore AS, et al. Household chemical exposures and the risk of canine malignant lymphoma, a model for human non-Hodgkin's lymphoma. Environ Res. 2012;112:171-6.

26. Gavazza A, Presciuttini S, Barale R, Lubas G, Gugliucci B. Association between canine malignant lymphoma, living in industrial areas, and use of chemicals by dog owners. J Vet Intern Med. 2001;15:190-5.

27. Pastor M, Chalvet-Monfray K, Marchal T, Keck G, Magnol JP, FournelFleury $C$, et al. Genetic and environmental risk indicators in canine non-Hodgkin's lymphomas: breed associations and geographic distribution of 608 cases diagnosed throughout France over 1 year. J Vet Intern Med. 2009;23:301-10.

28. Watanabe T, Sugiura T, Manabe S, Takasaki W, Ohashi Y. Low glutathione Stransferase dogs. Arch Toxicol. 2004;78:218-25.

29. Ginn J, Sacco J, Wong YY, Motsinger-Reif A, Chun R, Trepanier LA. Positive association between a glutathione-S-transferase polymorphism and lymphoma in dogs. Vet Comp Oncol. 2014;12:227-36.

30. Barrett JC, Fry B, Maller J, Daly MJ. Haploview: analysis and visualization of LD and haplotype maps. Bioinformatics. 2005;21:263-5.

31. Gaunt TR, Rodriguez S, Zapata C, Day IN. MIDAS: software for analysis and visualisation of interallelic disequilibrium between multiallelic markers. BMC Bioinformatics. 2006;7:227.

32. Sievers F, Wilm A, Dineen D, Gibson TJ, Karplus K, Li W, et al. Fast, scalable generation of high-quality protein multiple sequence alignments using Clustal Omega. Mol Syst Biol. 2011;7:539.

33. Ettwiller L, Paten B, Souren M, Loosli F, Wittbrodt J, Birney E. The discovery, positioning and verification of a set of transcription-associated motifs in vertebrates. Genome Biol. 2005;6:R104.

34. Lee $\mathrm{C}$, Huang $\mathrm{CH}$. LASAGNA-Search: an integrated web tool for transcription factor binding site search and visualization. BioTechniques. 2013:54:141-53.

35. Zuker M. Mfold web server for nucleic acid folding and hybridization prediction. Nucleic Acids Res. 2003;31:3406-15.

36. Legendre M, Pochet N, Pak T, Verstrepen KJ. Sequence-based estimation of minisatellite and microsatellite repeat variability. Genome Res. 2007;17:1787-96.

37. Tirosh I, Barkai N, Verstrepen KJ. Promoter architecture and the evolvability of gene expression. J Biol. 2009;8:95.

38. Landry CR, Lemos B, Rifkin SA, Dickinson WJ, Hartl DL. Genetic Properties Influencing the Evolvability of Gene Expression. Science. 2007;317:118-21.

39. Lindblad-Toh K, Wade CM, Mikkelsen TS, Karlsson EK, Jaffe DB, Kamal M, et al. Genome sequence, comparative analysis and haplotype structure of the domestic dog. Nature. 2005;438:803-19.

40. Vonholdt BM, Pollinger JP, Lohmueller KE, Han E, Parker HG, Quignon P, et al. Genome-wide SNP and haplotype analyses reveal a rich history underlying dog domestication. Nature. 2010;464:898-902.

41. Sutter NB, Eberle MA, Parker HG, Pullar BJ, Kirkness EF, Kruglyak L, et al. Extensive and breed-specific linkage disequilibrium in Canis familiaris. Genome Res. 2004;14:2388-96.

42. Parker HG, Kim LV, Sutter NB, Carlson S, Lorentzen TD, Malek TB, et al. Genetic structure of the purebred domestic dog. Science. 2004;304:1160-4.

43. Lohi H, Young EJ, Fitzmaurice SN, Rusbridge C, Chan EM, Vervoort M, et al. Expanded repeat in canine epilepsy. Science. 2005;307:81.

44. Pearson CE, Nichol Edamura K, Cleary JD. Repeat instability: mechanisms of dynamic mutations. Nat Rev Genet. 2005;6:729-42.

45. Hoogendoorn B, Coleman SL, Guy CA, Smith K, Bowen T, Buckland PR, et al. Functional analysis of human promoter polymorphisms. Hum Mol Genet. 2003;12:2249-54.

46. Verkerk AJ, Pieretti M, Sutcliffe JS, Fu YH, Kuhl DP, Pizzuti A, et al. Identification of a gene (FMR-1) containing a CGG repeat coincident with a breakpoint cluster region exhibiting length variation in fragile $X$ syndrome. Cell. 1991:65:905-14.

47. Grabczyk E, Kumari D, Usdin K. Fragile X syndrome and Friedreich's ataxia: two different paradigms for repeat induced transcript insufficiency. Brain Res Bull. 2001;56:367-73.

48. Roberts RL, Gearry RB, Bland MV, Sies CW, George PM, Burt M, et al. Trinucleotide repeat variants in the promoter of the thiopurine $S$ methyltransferase gene of patients exhibiting ultra-high enzyme activity. Pharmacogenet Genomics. 2008;18:434-8.
49. Johnson AC, Jinno Y, Merlino GT. Modulation of epidermal growth factor receptor proto-oncogene transcription by a promoter site sensitive to S1 nuclease. Mol Cell Biol. 1988;8:4174-84.

50. Whetstine JR, Witt TL, Matherly LH. The human reduced folate carrier gene is regulated by the AP2 and sp1 transcription factor families and a functional 61-base pair polymorphism. J Biol Chem. 2002;277:43873-80.

51. Contente A, Dittmer A, Koch MC, Roth J, Dobbelstein M. A polymorphic microsatellite that mediates induction of PIG3 by p53. Nat Genet. 2002;30: 315-20.

52. Yang L, Han Y, Suarez Saiz F, Minden MD. A tumor suppressor and oncogene: the WT1 story. Leukemia. 2007;21:868-76.

53. Ullmark T, Järvstråt $L$, Sandén $C$, Montano $G$, Jernmark-Nilsson H, Lilljebjörn $\mathrm{H}$, et al. Distinct global binding patterns of the Wilms tumor gene 1 (WT1) -KTS and +KTS isoforms in leukemic cells. Haematologica. 2017;102:336-45.

54. Roth C, Schuierer M, Günther K, Buettner R. Genomic structure and DNA binding properties of the human zinc finger transcriptional repressor AP2rep (KLF12). Genomics. 2000;63:384-90.

55. van Ham SM, van Alphen L, Mooi FR, van Putten JP. Phase variation of H influenzae fimbriae: transcriptional control of two divergent genes through a variable combined promoter region. Cell. 1993;73:1187-96.

56. Galvao R, Mendes-Soares L, Camara J, Jaco I, Carmo-Fonseca M. Triplet repeats, RNA secondary structure and toxic gain-of-function models for pathogenesis. Brain Res Bull. 2001;56:191-201.

\section{Submit your next manuscript to BioMed Central and we will help you at every step:}

- We accept pre-submission inquiries

- Our selector tool helps you to find the most relevant journal

- We provide round the clock customer support

- Convenient online submission

- Thorough peer review

- Inclusion in PubMed and all major indexing services

- Maximum visibility for your research

Submit your manuscript at www.biomedcentral.com/submit

) BioMed Central 